\title{
Application of alizarin colorimetric measurements for quantification of amine extraction by model food simulants from epoxy polymer
}

Tomasz Jeliński ${ }^{1 *}$, Piotr Cysewski ${ }^{1,2}$ and Edwin Makarewicz ${ }^{2}$

\begin{abstract}
A simple and straightforward method has been proposed for quantification of residual amine in cured epoxy resin. Non-bounded triethylenetetramine was extracted from epoxy polymer and determined via spectrophotometry using alizarin chromophore. Four solvents commonly used as food simulants, namely water, 95\% ethanol, 10\% ethanol and 3\% acetic acid were examined. Released amine induces changes in the absorption spectrum of alizarin, by decreasing the intensity of the maximum at $430 \mathrm{~nm}$ band and mutually increasing the $527 \mathrm{~nm}$ band. These changes were proportional to the amounts of amine concentration in samples. The statistical significance of obtained calibration curves was validated. Among studied solvents, the highest amine release was observed for water solution and $3 \%$ acetic acid, that is approximately $7 \% \mathrm{w} / \mathrm{w}$. The maximal amount of residual amine extracted with $95 \%$ ethanol was about $1.25 \%$, while for $10 \%$ ethanol this amount was $2 \%$. The effect of aging of the samples and exposure to artificial sunlight were also examined. The proposed method has been proven to be fast, low cost and directly applicable for analysis of typical epoxy resins.
\end{abstract}

Keywords: Epoxy resin; Alizarin; Triethylenetetramine; Food simulant; Residual amine; Curing

\section{Introduction}

Epoxy resins are widely applied as coatings, adhesives, construction composites etc. (Muskopf and McCollister 2002; Petrie 2006; Wicks et al. 2007). Common curing agents used with epoxy resins are aliphatic and aromatic polyamines (Petrie 2006; Prolongo et al. 2003; Wicks et al. 2007). However, the reaction of amine groups with oxirane rings is never complete, because the conversion degree is restricted by resin vitrification and therefore, residual amine hardeners can migrate from the cured polymer. This may cause serious problems due to the known toxicity of aliphatic and aromatic amines (Hughes et al. 1995; Patnaik 2007; Smith 2010). There are several examples of contact dermatitis resulting from the use of amine compounds in modified polymers (Conde-Salazar et al. 2004; Soto et al. 1989), and also the health problems caused by amine hardeners in epoxy systems have been studied shortly after the introduction

\footnotetext{
*Correspondence: tomasz.jelinski@cm.umk.pl

'Department of Physical Chemistry, Collegium Medicum, Nicolaus

Copernicus University, Kurpińskiego 5, 85-950 Bydgoszcz, Poland

Full list of author information is available at the end of the article
}

of epoxy resins into modern industry (Bourne et al. 1959). After many years of usage of epoxy resins in different industrial and commercial applications, the problem of health hazards caused by such systems is still not completely examined, although many published works have focused on allergies, caused by both the epoxy resin itself (Akita et al. 2001; Kumar and Freeman 1999; Sasseville 1998) as well as resin modifiers, such as hardeners (Bachanek et al. 2005; Bray 1999). For that reason, determination of non-bounded amine hardener is an important analytical task. Chromatographic methods are the ones mostly used (Dopico-Garcia et al. 2010; Paseiro-Cerrato et al. 2010) however, gas chromatography has limited applicability because of low volatility of multifunctional amines. Near-infrared spectroscopy is a convenient and rapid technique offering reliable assessments of amine conversion degree (Escola et al. 2005; Prolongo et al. 2003). Direct spectrophotometry and spectrofluorimetry are rather less applicable to aliphatic amines, because they do not possess characteristic UV-VIS absorbance or fluorescence properties. On the other hand, both the techniques became fully applicable 
when appropriate colored indicator is used (Ajayakumar and Mukhopadhyay 2009; Bao et al. 2010; Basurto et al. 2005; Comes et al. 2004; García-Acosta et al. 2006; Liu et al. 2006; Oliveri and Di Bella 2011; Staneva et al. 2007). One of the promising indicators for low-polar solvents is alizarin (1,2-dihydroxy-9,10-anthraquinone) (del Río et al. 2010). Anthraquinone derivatives in general are known as analytical reagents (Hosseini and Asadi 2009; Mitic et al. 2007). Alizarin itself is applied in chemical analysis as well (Chamsaz et al. 2000; Feng et al. 2011). Alizarin exists in three forms of different color, namely the protonated form and two deprotonated forms corresponding to the monoanion and dianion (Das et al. 2002; Cysewski et al. 2012; Preat et al. 2009). The absorption maximum of the neutral non-dissociated form is located at $430 \mathrm{~nm}$ (Preat et al. 2009; Say-LiangFat and Cornard 2011) and the monoanionic form is characterized by an absorption maximum at around $530 \mathrm{~nm}$ (Quinti et al. 2003; Savko et al. 2007). The color change of alizarin can be modeled quite accurately based on simple protocol within TD-DFT framework, which has been shown by us in one of our earlier studies (Cysewski et al. 2012). This makes the use of alizarin chromophore very convenient since it is sensitive to the presence of proton acceptor agents even in non-water solutions.

\section{Experimental}

\section{Reagents and chemicals}

Mid-viscosity epoxy resin with trade name Epidian 5 (Cedar, Poland) was used in the studies. Triethylenetetramine (TETA), known as one of the most widely used hardeners (Czub 2009; Manthey et al. 2011; Roskowicz and Smal 2011; Tripathy et al. 2011) has been purchased also from Cedar under trade name Z1. The above compounds were used without further purification. Analytical grade ethanol and acetic acid were supplied by POCH S.A. (Poland). Alizarin has been supplied by Sigma-Aldrich.

\section{Procedure}

Stock solutions of TETA in widely accepted food simulants such as water, 95\% ethanol, 10\% ethanol and 3\% acetic acid were prepared with the concentration of $0.160 \mathrm{mg} / \mathrm{ml}$ each. A series of diluted solutions was then prepared by adding different volumes of the solvent to a standard volume of the stock solution. Alizarin stock solutions in 95\% ethanol, 10\% ethanol, 3\% acetic acid and methanol were prepared with the concentration of $0.4685 \mathrm{mmol} / \mathrm{l}$. Aqueous solution was not used because the solubility of alizarin in water is very limited. Calibration samples were prepared by mixing $1.5 \mathrm{~cm}^{3}$ of each diluted amine solution with $1.5 \mathrm{~cm}^{3}$ of alizarin stock solution directly in a spectrophotometric cuvette. In such a way, three sets of calibration curves were obtained in water-methanol (50\% v/v), 95\% ethanol and 10\% ethanol solutions. For $3 \%$ acetic acid, the alizarin stock solution was alkalized to $\mathrm{pH}$ equal to 12 and then the acidic solution of TETA was added as described above. Absorption spectra were recorded using a single-beam UV/VIS spectrophotometer (Merck, Spectroquant Pharo 300) with a wavelength resolution of $1 \mathrm{~nm}$.

Series of epoxy polymer samples has been prepared at the hardener content equal to $8,10,12,14$ and $16 \mathrm{phr}$. Pre-weighted amounts of the epoxy resin and TETA were thoroughly mixed and left for 24 hours at ambient temperature for the completion of the curing process. Cured polymer pellets of about $50 \mathrm{mg}$ were weighed and placed in sealed tubes filled with $3 \mathrm{ml}$ of solvent for extraction. Preliminary tests showed that the extraction process is complete after 3 hours, which is documented in the Appendix. Next, $1.5 \mathrm{ml}$ of the extract was taken and put into a cuvette containing $1.5 \mathrm{ml}$ of the alizarin stock solution (alkalized in the case of $3 \%$ acetic acid solution). Then, the spectra were recorded in the same way as in the case of the calibration curves.

\section{Aging effect and sunlight exposure}

In order to investigate the effect of aging of the samples on the amine release process, one series of the samples was examined immediately after preparation, while the pellets from the second series were left for six months in room temperature and then examined.

An artificial sunlight lamp was used for simulating the effect of sunlight exposure. The power density of this lamp was measured as a function of the length from the lamp to the surface containing the examined epoxy pellets. Also, the temperature was measured in order to avoid overheating of the samples. The length of $12.5 \mathrm{~cm}$ was chosen, giving a power density of $9.2 \mathrm{~kW} / \mathrm{m}^{2}$. Since the annual solar irradiance in middle Europe (e.g. Poland) is about $1000 \mathrm{kWh} / \mathrm{m}^{2}$, a series of time intervals from 1 to 100 hours mimicked the amount of energy distributed to the samples throughout the one year span.

\section{Statistical analysis}

Statistical analysis has been conducted as follows. Linear equations and R2 coefficients were used to determine the variance of the $y$-intercept (denoted as $\mathrm{C}$ ).

The variance of the slope coefficient (denoted as B) was calculated according to the formula:

$$
\operatorname{Var}(B)=\frac{B^{2} \cdot \phi^{2}}{R^{2} \cdot f}
$$

where $f$ is the number of degrees of freedom (for $\alpha=$ 0.05 the $f$ parameter equals 3 ) and $\phi^{2}$ is the indetermination coefficient calculated as follows: 


$$
\phi^{2}=\frac{\sum_{i=1}^{N D}\left(Y_{i}-Y_{i, \text { calc }}\right)^{2}}{\sum_{i=1}^{N D}\left(Y_{i}-\bar{Y}_{i}\right)^{2}=1-R^{2}}
$$

where $Y_{i}$ are the experimentally obtained values and $Y_{i, \text { calc }}$ are the ones estimated on the basis of the obtained linear equation.

The variance of the y-intercept is connected with variance of the slope coefficient with a simple dependence:

$$
\operatorname{Var}(\mathrm{C})=\operatorname{Var}(\mathrm{B}) \cdot \overline{\mathrm{X}}^{2}
$$

The standard deviation of $y$-intercept is then calculated as the square root of the variance:

$$
\mathrm{SD}(\mathrm{C})=\sqrt{\operatorname{Var}(\mathrm{C})}
$$

The limit of detection (LOD) and the limit of quantification (LOQ) where then calculated with the following equations:

$$
\begin{aligned}
\mathrm{LOD} & =\frac{3.3 \cdot S D(C)}{B} \\
\mathrm{LOQ} & =3 \cdot \mathrm{LOD}
\end{aligned}
$$

\section{Results and discssion \\ Calibration curves}

Addition of increased amounts of TETA to alizarin solution results in substantial changes of UV-VIS spectra. The absorption maximum of alizarin at $430 \mathrm{~nm}$ is decreased and the height of the peak at $527 \mathrm{~nm}$ is increased. It is obvious, that the observed spectral changes are caused by the increase of solution alkalinity and the formation of alizarin amine complex with pseudodissociation to anionic form. This is discussed in the Appendix section. These regularities related to color change from colorless to magenta-red are observed in all of the solvents studied.

The absorbance values at the wavelengths $527 \mathrm{~nm}$ (Figure 1) and $430 \mathrm{~nm}$ (Appendix) as well as the absorbance ratio (Appendix) were taken into account and tested as potential calibration curves for amine determination. The statistical analysis showed that the calibration curve corresponding to the absorbance values at $527 \mathrm{~nm}$ is the most reliable and was used in further analysis.

The obtained linear equations, determination coefficients, values of limits of detection (LOD) and quantification (LOQ) for this curve have been presented in Table 1, while for the two other curves in the Appendix.

From the data provided in Table 1 and Appendix one can see that the absorbance values at $527 \mathrm{~nm}$ proved to have the best linearity among the three curves. Although the curve utilizing the ratio of absorbencies has a slightly better linearity in the case of $10 \%$ ethanol and 3\% acetic acid, it performs much worse in the case of $95 \%$ ethanol and water. On the other hand, the curve utilizing the absorbance values at $430 \mathrm{~nm}$ has a good linearity for $95 \%$ ethanol and water, although worse than the ' $527 \mathrm{~nm}$ ' curve, but it performs much worse in the case of $10 \%$ ethanol and 3\% acetic acid. The above observations are also confirmed by the LOD and LOQ values for these curves. Also, the solvents used influenced both the detection and quantification limits. The best sensitivity is obtained in the case of $3 \%$ acetic acid, while the worst

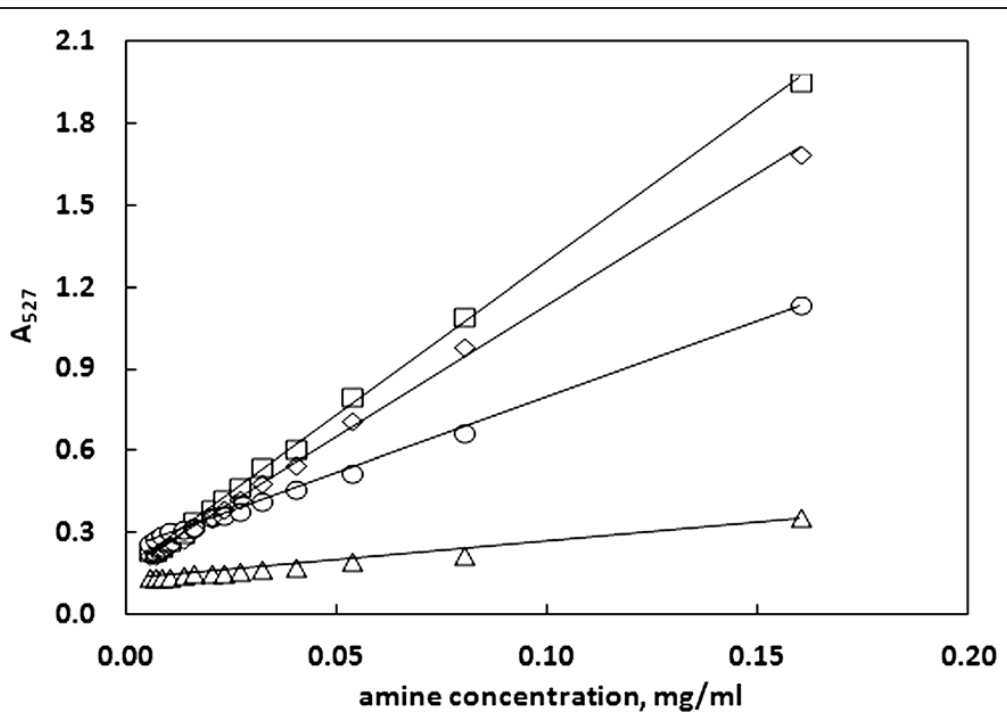

Figure 1 The absorbance values at $\mathbf{5 2 7} \mathrm{nm}$ as a function of amine concentration. The calibration plots are obtained in water-methanol $(\diamond), 95 \%$ ethanol $(0), 10 \%$ ethanol $(\Delta)$ and $3 \%$ acetic acid $(\square)$ solutions. 
Table 1 Linear equations, $R^{2}$ coefficients, values of the standard deviation of $y$-intercept (SD(A)) and the values of limits of detection (LOD) and quantification (LOQ) for the calibration curve utilizing the absorbance at $\mathbf{5 2 7} \mathbf{\mathrm { nm }}$

\begin{tabular}{lllll}
\hline Calibration data & $\mathbf{9 5 \%}$ ethanol & $\mathbf{1 0 \%}$ ethanol & $\mathbf{3 \%}$ acetic acid & Water \\
\hline $\mathrm{SD}(\mathrm{C})$ & 0.000205 & 0.000334 & 0.000621 & 0.000596 \\
LOD $[\mathrm{mg} / \mathrm{ml}]$ & 0.000122 & 0.000795 & 0.000101 & 0.000203 \\
LOQ $[\mathrm{mg} / \mathrm{ml}]$ & 0.000366 & 0.002385 & 0.000304 & 0.000610 \\
$\mathrm{R}^{2}$ coefficient & 0.9988 & 0.9922 & 0.999 & 0.998 \\
Linear equation & $y=5.544 x+0.242$ & $y=1.389 x+0.128$ & $y=11.293 x+0.1644 \quad$ & $y=9.6858 x+0.165$ \\
\hline
\end{tabular}

sensitivity characterizes $10 \%$ ethanol, although the limits of detection and quantification are satisfactory for all the solvents. One can infer, that the most appropriate calibration curve for TETA determination is provided with the absorbance at $527 \mathrm{~nm}$. Besides, all four food simulant solvents may be used for analytical purpose, with conjunction of alizarin chromophore.

\section{Quantification of amine extraction}

The cured epoxy resin pellets, both freshly prepared and left for six months in dark and ambient conditions, were extracted by four different solvents and the amount of amine released was determined using the calibration curve utilizing the absorbance at $527 \mathrm{~nm}$. The obtained results are presented in Figure 2. Linear dependence between the residual amine amount in cured epoxy resin and the initial amine amount is observed with all the solvents applied. The polarity of the solvent affected the amount of amine extracted from the polymer. For samples examined immediately after the curing process the amount of released amine varies from $0.35 \%$ to $1.25 \%$ in the case of $95 \%$ ethanol, from $3 \%$ to $4 \%$ in the case of $10 \%$ ethanol, from $5.5 \%$ to $6.5 \%$ in the case of water solution and from $6 \%$ to $7.25 \%$ in the case of $3 \%$ acetic acid. For the samples that were left for six months the amounts of released amine were smaller. Measurements for 10 pellets containing the same amount of amine, both fresh and left for six months, were performed for water and 95\% ethanol. Interestingly, statistically significant differences between the two series of pellets were noticed in the case of $95 \%$ ethanol solution, while in the case of water solution, these differences were negligible. The results obtained for pellets containing $12 \mathrm{phr}$ of amine are also presented in Figure 2. In the case of $95 \%$

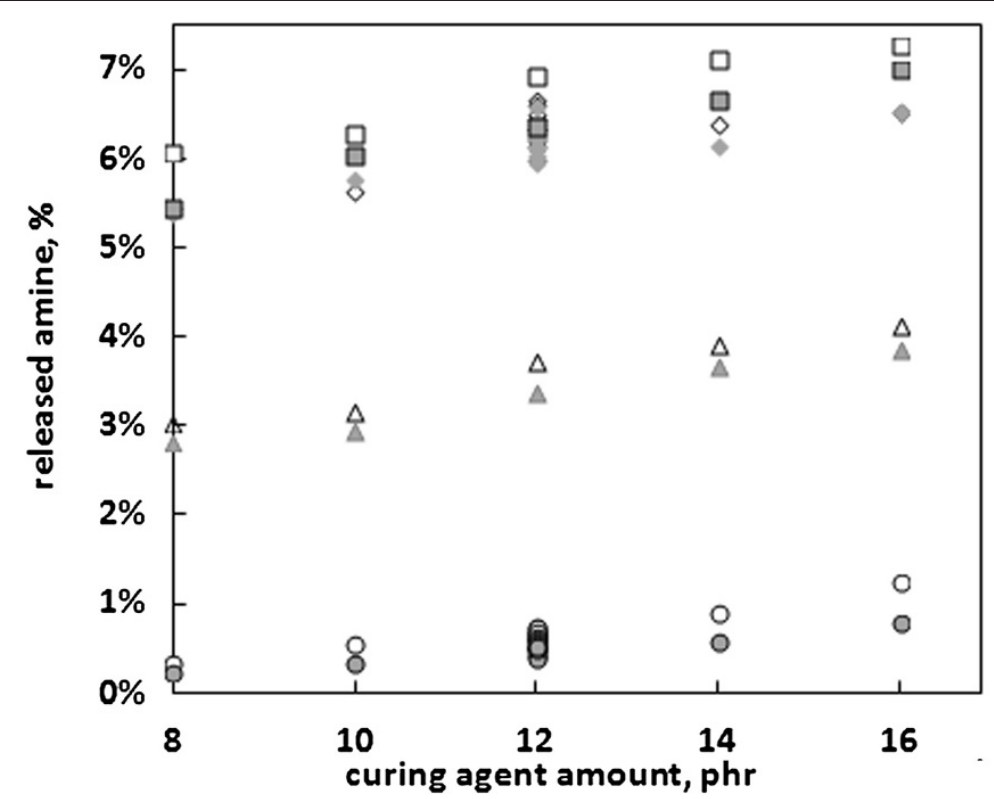

Figure 2 Amounts of residual amine extracted from cured epoxy resin with water $(\diamond), 95 \%$ ethanol $(0), 10 \%$ ethanol $(\Delta)$ and $3 \%$ acetic acid ( $\square$ ). The determinations performed using calibration with $527 \mathrm{~nm}$ absorbance for pellets after 24 hours of curing (white)and pellets after six months in room temperature (grey). 
ethanol the standard deviations were $0.5 \%$ and $0.6 \%$ for freshly prepared pellets and those left for six months, respectively. The statistical significance was estimated based on P values, obtained using two-way heteroscedastic statistical test (T-test). In the case of water the standard deviations were $0.17 \%$ and $0.21 \%$, while conducting the same test leads to the conclusion that the differences between populations are not statistically important.

The polarity of TETA results in very high affinity towards water, which was documented by extraction experiments. Having the above in mind, the lack of differences in the amounts of released amine for fresh samples and samples aging for six months suggests that there was no further progress in cross-linking reaction during this period of time. However, it is interesting to see, that such statistically significant differences occur in the case of much less polar 95\% ethanol solution. This may suggest that during the aging of the sample partial penetration of the amine inside the polymer takes place. However, this physical process leads to such a small change of affinity towards water, that the extraction with the use of this polar solvent causes the release of all, or at least the majority, of the unbounded amine. Also, it can be suggested that the amine sorption process is caused by intermolecular interactions weaker than those stabilizing amine solutions in water, but stronger than those of TETA-ethanol system. Thus, residual amounts of amine that are not chemically bounded can be released to water. Also, wet surfaces coated with epoxy polymers are probably rich in extracted portions of amine, which poses some ecological challenge if such polymers are used. On the other hand, this releasing process occurs only for limited time period that is restricted to total amount of free amine in samples. Since water is a very effective extraction media it seems to be rational to propose a post-polymerization process of immersion in water as a natural way of freeing the polymer from the residual amine.

The results of the exposure of the cured epoxy pellets to artificial sunlight are presented in Figure 3. As it could be expected, the increased temperature and UV radiation induced a progress in the cross-linking reaction. This was documented by the decrease in the amounts of extracted residual amine. When plotting the amount of extracted amine against the irradiance of the light source, one can see its exponential decrease. In the case of water and 3\% acetic acid, the amount of released amine with the maximum irradiance used was about $1.2 \%$, which is more than 5 times less than for the samples that did not undergo light exposure. In the case of $10 \%$ ethanol solution the minimal amount reached $0.5 \%$, while in the case of $95 \%$ ethanol the released amount of amine with the maximum irradiance could not be determined and the smallest determinable amounts of amine were extracted with $55 \mathrm{kWh} / \mathrm{m}^{2}$. This effect was accompanied by a change of color of the epoxy pellets, which gradually turned yellow. This color change shows that the ecologically valuable decrease in

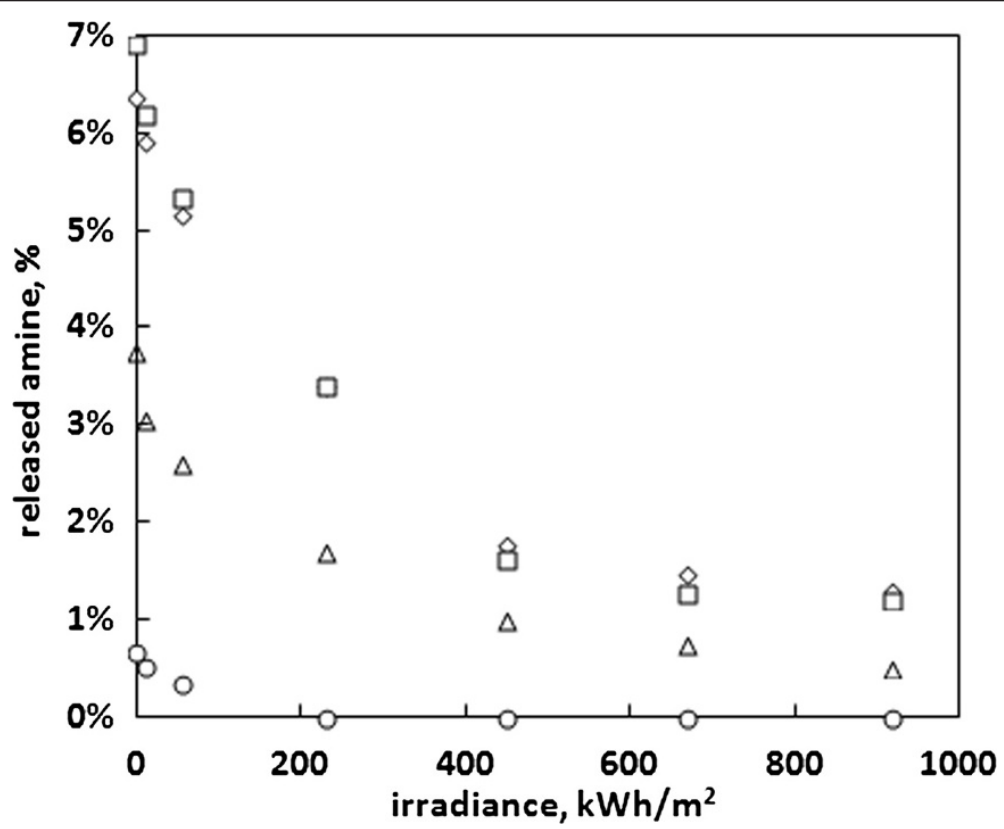

Figure 3 Amounts of residual amine extracted from cured epoxy resin after exposure to artificial sunlight with water $(\diamond), 95 \%$ ethanol $(0), 10 \%$ ethanol $(\Delta)$ and $3 \%$ acetic acid $(\square)$ as the function of the irradiance of the light source. 
the amount of released amine may not come in pair with the desired mechanical or technological properties of the epoxy polymer.

\section{Conclusions}

A new, simple and accurate method of determination of residual amine in epoxy polymer has been developed. The method utilizes the susceptibility of alizarin to undergo color changes in proton accepting media. THETA, acting as a base, induces changes in the alizarin chromophore which enables the use of spectrometric methods in quantitative determinations. The calibration plot corresponding to the absorbance at $527 \mathrm{~nm}$ was identified as being particularly suitable for determination of residual amine amount. The sensitivity of the developed method is fully adequate for typical epoxy resin formulations. It has been shown that the quantity of released amine is the largest in the case of water and 3\% acetic acid(reaching 7\%) and the smallest in the case of 95\% ethanol (slightly above 1\%). Such results indicate that the amounts of released amine are relatively large and therefore the release of amine from the epoxy polymer is ecologically important, since amines create significant health hazards for humans, such as dermatitis. The post-polymerization immersion of epoxy polymer in water may be used as a simple method of freeing the polymer from the residual amine. The proposed analytical method proved its usefulness in examining two different effects, namely the aging of the samples and their exposure to artificial sunlight. It was also demonstrated that the aging of the samples causes a statistically important difference in the residual amine extraction for $95 \%$ ethanol but no such difference can be observed in the case of water. This might suggest, that there is no further progress in cross-linking reaction during this time and the nature of this effect is only diffusional. Also, the exposure of the samples to artificial sunlight caused a progress in the cross-linking reaction and a resulting decrease in the amounts of extracted residual amine.

\section{Appendix}

\section{Extraction time measurements}

Before the quantification of amine release it was necessary to determine the time in which the extraction of cured epoxy pellets is complete. Therefore, the pellets were extracted in all the solvents for different time periods and the obtained results are shown in Figure 4. A gradual increase of the amount of extracted amine was observed during time but after 120 minutes of extraction the amount of extracted amine remained unchanged despite the increasing extraction time. Therefore, an optimal value of 3 hours has been chosen as the extraction time.

\section{Calibration curves less suitable for amine determination}

The calibration curve corresponding to the absorbance at $527 \mathrm{~nm}$ wavelength was chosen as the most appropriate one for residual amine determination because of its

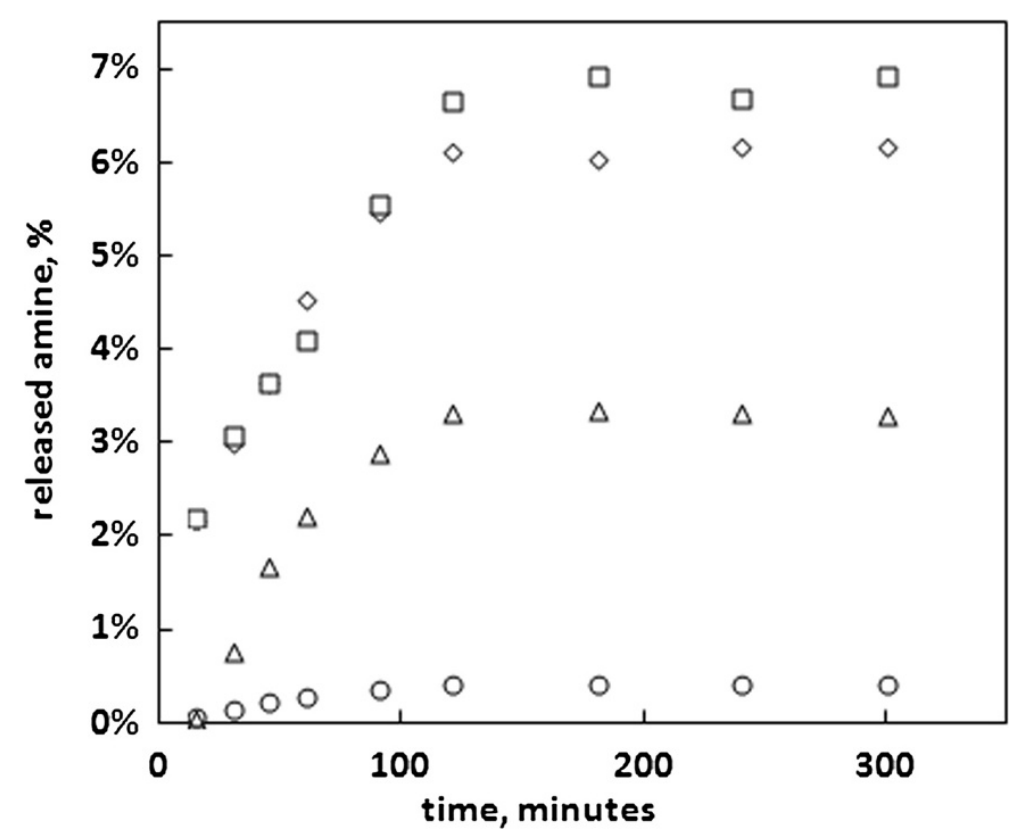

Figure 4 The amine release as a function of extraction time, for water-methanol $(\diamond), 95 \%$ ethanol $(0), 10 \%$ ethanol $(\Delta)$ and $3 \%$ acetic acid ( $\square$ ) solutions. 


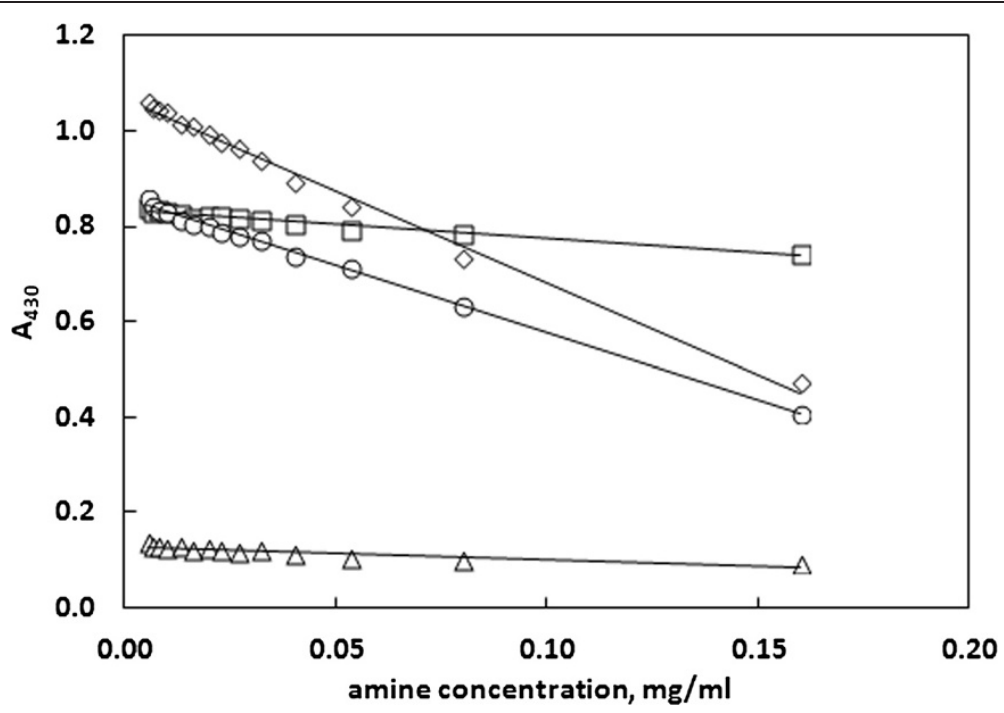

Figure 5 The absorbance values at $\mathbf{4 3 0} \mathrm{nm}$ as a function of amine concentration. The calibration plots are obtained in water-methanol $(\diamond), 95 \%$ ethanol $(0), 10 \%$ ethanol $(\Delta)$ and $3 \%$ acetic acid $(\square)$ solutions.

good linearity in all the solvents and satisfactory values of detection and quantification limits. However, the two other considered curves also performed well in some cases. The curve utilizing absorbance values at $430 \mathrm{~nm}$ wave length is suitable for all the solvents except $10 \%$ ethanol, while the curve utilizing the ratio of absorbencies at $430 \mathrm{~nm}$ and $527 \mathrm{~nm}$ wavelengths is less suitable for $95 \%$ ethanol. The two considered here calibration curves are presented in Figure 5 and Figure 6. The obtained linear equations, determination coefficients, values of limits of detection (LOD) and quantification
(LOQ) for these curves have been presented in Table 2 and Table 3.

\section{The $\mathrm{pH}$ values of Alizarin with TETA stock solutions}

The $\mathrm{pH}$ value of the alizarin-TETA system is mostly dependant on the amine concentration. Since alizarin has hydroxyl groups in its structure, in water it undergoes reaction (7):

$$
\mathrm{AZ}-\mathrm{OH}+\mathrm{H}_{2} \mathrm{O} \leftrightarrow \mathrm{AZ}^{-} \mathrm{O}^{-}+\mathrm{H}_{3} \mathrm{O}^{+}
$$

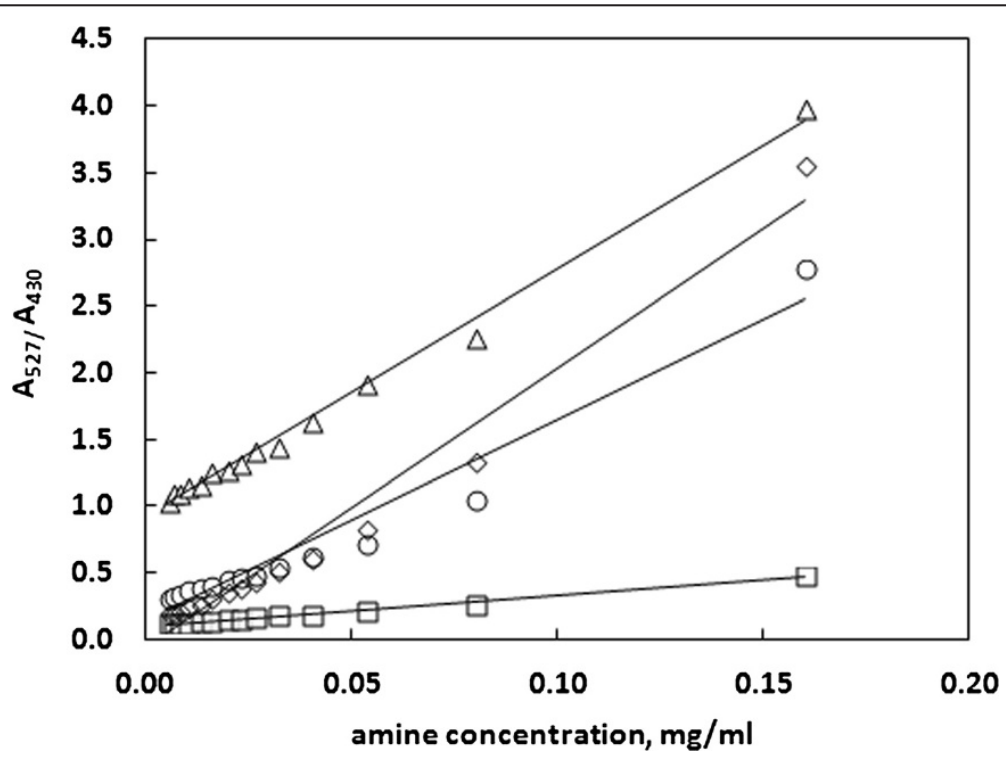

Figure 6 The values of the absorbencies ratio as a function of amine concentration. The calibration plots are obtained in water-methanol $(\diamond), 95 \%$ ethanol $(0), 10 \%$ ethanol $(\Delta)$ and $3 \%$ acetic acid $(\square)$ solutions. 
Table 2 Linear equations, $R^{2}$ coefficients, values of the standard deviation of $y$-intercept (SD(A)) and the values of limits of detection (LOD) and quantification (LOQ) for the calibration curve utilizing the absorbance at $430 \mathrm{~nm}$

\begin{tabular}{lllll}
\hline Calibration data & $\mathbf{9 5 \%}$ ethanol & $\mathbf{1 0 \%}$ ethanol & $\mathbf{3 \%}$ acetic acid & Water \\
\hline SD $(\mathrm{C})$ & 0.000217 & 0.001330 & 0.000484 & 0.000857 \\
LOD $[\mathrm{mg} / \mathrm{ml}]$ & 0.000254 & 0.016130 & 0.002612 & 0.000733 \\
LOQ $[\mathrm{mg} / \mathrm{ml}]$ & 0.000763 & 0.048400 & 0.007838 & 0.002201 \\
$R^{2}$ coefficient & 0.9975 & 0.8532 & 0.9746 & 0.992 \\
Linear equation & $y=-2.823 x+0.860$ & $y=-0.272 x+0.127$ & $y=-0.612 x+0.837$ & $y=-3.857 x+1.068$ \\
\hline
\end{tabular}

Table 3 Linear equations, $R^{2}$ coefficients, values of the standard deviation of $y$-intercept (SD(A)) and the values of limits of detection (LOD) and quantification (LOQ) for the calibration curve utilizing the ratio of absorbencies

\begin{tabular}{lllll}
\hline Calibration data & 95\% ethanol & 10\% ethanol & 3\% acetic acid & Water \\
\hline SD $(\mathrm{C})$ & 0.020758 & 0.003153 & 0.000619 & 0.019432 \\
LOD $[\mathrm{mg} / \mathrm{ml}]$ & 0.004527 & 0.000560 & 0.000979 & 0.003051 \\
LOQ $[\mathrm{mg} / \mathrm{ml}]$ & 0.013582 & 0.001680 & 0.002938 & 0.009154 \\
$R^{2}$ coefficient & 0.9564 & 0.9945 & 0.9904 & 0.97 \\
Linear equation & $y=15.131 \mathrm{x}+0.131$ & $y=18.583 x+0.9191$ & $y=2.0863 x+0.1079 \quad$ & $y=21.016 x-0.0621$ \\
\hline
\end{tabular}

Therefore, is not surprising that slightly acidic $\mathrm{pH}$ values are observed. When the amine is added reaction (8) takes place:

$$
\mathrm{R}-\mathrm{NH}_{2}+\mathrm{H}_{2} \mathrm{O} \leftrightarrow \mathrm{R}-\mathrm{N}^{+} \mathrm{H}_{3}+\mathrm{OH}^{-}
$$

This reaction is the source of hydroxide ions, the presence of which shifts the balance of reaction (7) towards

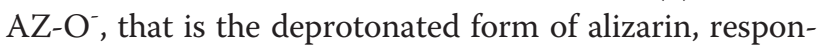
sible for the color change of the alizarin solution. This can be observed in Figure 7. Similar conclusions can be drawn for other non-acidic solutions, as methanol or acetone.

Quantification of amine release from 3\% acetic acid

In the case of $3 \%$ acetic acid, the initial solution of alizarin was alkalized to $\mathrm{pH}=12$ since alizarin is not sensitive in the range of $\mathrm{pH}$ characterizing acidic solutions. Still, the system is sensitive to changes of amine amount, as it was demonstrated in Figure 7. Unfortunately, artificial rise of $\mathrm{pH}$ unables the direct determination of amine amount based on spectroscopic response of alizarin chromophore. In this situation, the system is much more

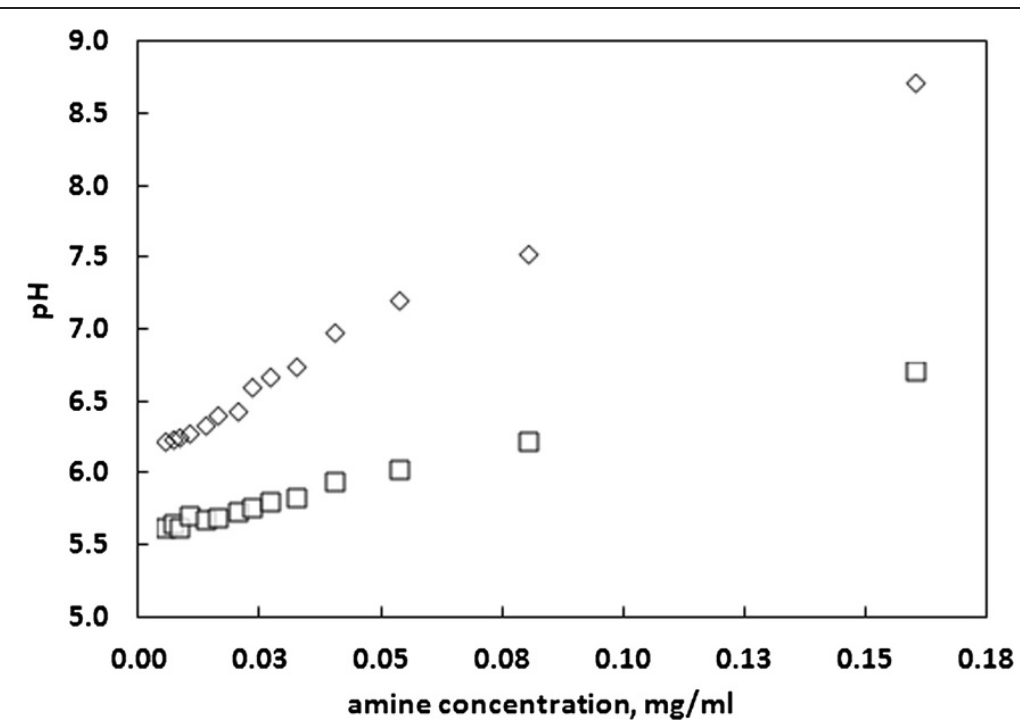

Figure $7 \mathrm{pH}$ values of diluted TETA stock solutions as a function of amine concentration, for water-methanol $(\diamond)$ and $3 \%$ acetic acid ( $\square$ ) solutions. 
complicated what can be described by the existence of the following equilibria:

$$
\begin{aligned}
& \mathrm{H}-\mathrm{Ac} \leftrightarrow \mathrm{Ac}^{-}+\mathrm{H}^{+} \\
& \mathrm{H}-\mathrm{AC}+\mathrm{OH}^{-} \leftrightarrow \mathrm{Ac}^{-}+\mathrm{H}_{2} \mathrm{O} \\
& \mathrm{H}-\mathrm{AC}+\mathrm{R}-\mathrm{NH}_{2} \leftrightarrow \mathrm{R}-\mathrm{N}^{+} \mathrm{H}_{3}+\mathrm{Ac}^{-} \\
& \mathrm{AZ}-\mathrm{OH}+\mathrm{H}_{2} \mathrm{O} \leftrightarrow \mathrm{AZ}^{-} \mathrm{O}^{-}+\mathrm{H}_{3} \mathrm{O}^{+}
\end{aligned}
$$

These additional equilibria can be interpreted in terms of several buffers formed by acetic acid/ $\mathrm{NaOH}$, acetic acid/ amine and with a lesser extent alizarin/amine and alizarin/ $\mathrm{NaOH}$. Despite a complicated interrelation between chemical species, there is a linear response of $\mathrm{pH}$ with respect to the added amount of TETA. The rise of amine concentration increases $\mathrm{AC}^{-}$concentration and at the same time shits the balance of reactions (9) and (10) toward reactants. Consequently, a decrease of acidity is associated with promotion of reaction (12), what can be observed by spectroscopic measurements. Thus, despite a complicated equilibriums system, it can be still used for quantification of amine concentration, taking into account the relative change of absorption bands of alizarin. For this purpose the calibration curve was modified according to the following formula:

$$
\begin{aligned}
& \mathrm{A}_{\mathrm{Ac}}(\lambda 527)=\mathrm{A}_{\mathrm{H} 2 \mathrm{O}}(\lambda 527)+\Delta \mathrm{A}_{\mathrm{Ac}}(\lambda 527) \\
& \Delta \mathrm{A}_{\mathrm{Ac}}=\mathrm{A}_{\mathrm{Ac}}\left(\lambda 527, \mathrm{C}_{\mathrm{x}}\right)-\mathrm{A}_{\mathrm{Ac}}\left(\lambda 527, \mathrm{C}_{0}\right)
\end{aligned}
$$

where: $A_{\mathrm{H} 2 \mathrm{O}}(\lambda 530)$ is the absorbance of water-methanol solution for given amine concentration; $\mathrm{A}_{\mathrm{Ac}}\left(\lambda 527, \mathrm{C}_{\mathrm{x}}\right)$ is the absorbance of the $3 \%$ acetic acid solution for the same amine concentration and $\mathrm{A}_{\mathrm{Ac}}\left(\lambda 527, \mathrm{C}_{0}\right)$ is the absorbance of the $3 \%$ acetic acid modified with $\mathrm{NaOH}$. This means that the relative change of absorption in the case of acetic acid solution is used instead of a direct one.

\section{Competing interests}

The authors declare that they have no competing interests.

\section{Authors' contributions}

TJ and PC designed, executed and analyzed the entire experiment and prepared the manuscript. EM assisted in the design and analysis of the experiment and in preparation of the manuscript. All authors read and approved the final manuscript.

\section{Author details}

${ }^{1}$ Department of Physical Chemistry, Collegium Medicum, Nicolaus Copernicus University, Kurpińskiego 5, 85-950 Bydgoszcz, Poland. ${ }^{2}$ Faculty of Chemical Technology and Engineering, Division of Materials Chemistry and Protective Coatings University of Technology and Life Sciences, Seminaryjna 3, 85-326 Bydgoszcz, Poland.

Received: 28 May 2013 Accepted: 23 October 2013

Published: 6 November 2013

\section{References}

Ajayakumar MR, Mukhopadhyay (2009) Naphthalene-bis-hydrazimide: radical anions and ICT as new bimodal probes for differential sensing of a library of amines. Chem Commun 25:3702-3704
Akita H, Washimi Y, Akamatsu H, Yoshimasa F, Kayoko M (2001) Erythemamultiforme-like occupational contact dermatitis due to bisphenol A. Contact Dermatitis 45:305

Bachanek T, Samborski D, Chalas R, Wolanska E (2005) Evaluation of teeth loss among workers in the laminate and composite materials department of aircraft factory. Agric Environ Med 12:325-329

Bao BQ, Yuwen LH, Zheng XN, Weng LX, Zhu XR, Zhan XW, Wang LH (2010) A fluorescent conjugated polymer for trace detection of diamines and biogenic polyamines. J Mater Chem 20:9628-9634

Basurto S, Torroba T, Comes M, Martínez-Máñez R, Sancenón F, Villaescusa LA Amorós P (2005) New Chromogenic Probes into Nanoscopic Pockets in Enhanced Sensing Protocols for Amines in Aqueous Environments. Org Lett 7:5459-5472

Bourne LB, Milner FJM, Alberman KB (1959) Health Problems of Epoxy Resins and Amine-curing Agents. Brit J Industr Med 16:81-97

Bray PG (1999) Epoxy resins. Occup Med 14:743-1786

Chamsaz M, Zavar MHA, Hosseini MS (2000) Flotation Spectrophotometric Determination of Aluminium with Alizarin. Anal Lett 33:1625-1633

Comes M, Marcos MD, Martínez-Máñez R, Sancenón F, Soto J, Villaescusa LA, Amorós P, Beltrán D (2004) Chromogenic Discrimination of Primary Aliphatic Amines in Water with Functionalized Mesoporous Silica. Adv Mater 16:1783-1786

Conde-Salazar L, Valks R, Acebes CG, Bertó J (2004) Occupational allergic contact dermatitis from antioxidant amines in a dental technician. Dermatitis 15(4):197-200

Cysewski P, Jelinski T, Przybylek M, Shyichuk A (2012) Color prediction from first principle quantum chemistry computations: a case of alizarin dissolved in methanol. New J Chem 36:1836-1843

Czub P (2009) Synthesis of high-molecular-weight epoxy resins from modified natural oils and Bisphenol A or BisphenolA-based epoxy resins. Polym Advan Technol 20:194-208

Das S, Bhattacharya A, Mandal PC, Rath MC, Mukherjee T (2002) One-electron reduction of 1,2-dihydroxy-9,10-anthraquinone and some of its transition metal complexes in aqueous solution and in aqueous isopropanol-acetone-mixed solvent: a steady-state and pulse radiolysis study. Radiat Phys Chem 65:93-100

del Río V, Larrech MS, Callao MP (2010) Sequential injection titration method using second-order signals: Determination of acidity in plant oils and biodiesel samples. Talanta 81:1572-1577

Dopico-Garcia MS, Lopez-Vilarino JM, Fernandez-Martinez G, Gonzalez-Rodriguez MV (2010) Liquid chromatography method to determine polyamines in thermosetting polymers. Anal Chim Acta 667:123-129

Escola MA, Moina CA, Nino Gómez AC, Ybarra GO (2005) The determination of the degree of cure in epoxy paints by infrared spectroscopy. Polym Test 24:572-575

Feng GE, Jiang L, Liu D, Chen C (2011) Interaction between alizarin and human serum albumin by fluorescence spectroscopy. Anal Sci 27:79-84

García-Acosta B, Comes M, Bricks JL, Kudinova MA, Kurdyukov W, Tolmachev Al, Descalzo AB, Marcos MD, Martínez-Máñez R, Moreno A, Sancenón F, Soto J, Villaescusa LA, Rurack K, Barat JM, Escriche I, Amorós P (2006) Sensory hybrid host materials for the selective chromo-fluorogenic detection of biogenic amines. Chem Commun 21:2239-2241

Hosseini MS, Asadi M (2009) Speciation determination of chromium using 1 4- diaminoanthraquinone with spectrophotometric and spectrofluorometric methods. Anal Sci 25:807-812

Hughes JS, Biddinger GR, Mones E (1995) Environmental Toxicology \& Risk Assessment. American Society for testing and Materials, Philadelphia

Kumar A, Freeman S (1999) Leukoderma following occupational allergic contact dermatitis. Contact Dermatitis 41:94-98

Liu C, Lin JT, Wang SH, Jiang JC, Lin LG (2006) Chromogenic calixarene sensors for amine detection. Sens Actuat B 108:521-527

Manthey NW, Cardona F, Aravinthan T, Cooney T (2011) Cure kinetics of an epoxidized hemp oil based bioresin system. J Appl Polym Sci 122:444-45

Mitic SS, Miletic GZ, Pavlovic AN, Tosic SB, Velimirovic DS (2007) Development and Evaluation of a Kinetic-Spectrophotometric Method for Determination of Arginine. J Chin Chem Soc 54:47-54

Muskopf JW, McCollister SB, Bohnet M, Brinker J, Comils B (2002) Ullmanns's encyclopedia of industrial chemistry. John Wiley and Sons, New York

Oliveri IP, Di Bella S (2011) Sensitive fluorescent detection and Lewis basicity of aliphatic amines. J Phys Chem 115:14325-14330

Paseiro-Cerrato R, Rodríguez-Bernaldo de Quirós A, Sendón R, Bustos J, Santillana MI, Cruz JM, Paseiro-Losada P (2010) Chromatographic Methods for the Determination of Polyfunctional Amines and Related Compounds Used as Monomers and Additives in Food Packaging Materials: A State-of-the-Art Review. Compr Rev Food Sci Food Saf 9(6):676-694 
Patnaik A (2007) A Comprehensive Guide to the Hazardous Properties of Chemical Substances. John Wiley and Sons, New York

Petrie EM (2006) Epoxy Adhesive Formulations. McGraw-Hill, New York

Preat J, Laurent A, Michaux C, Perpete E, Jacquemin D (2009) Impact of tautomers on the absorption spectra of neutral and anionic alizarin and quinizarin dyes. J Mol Struct (Theochem) 901:24-30

Prolongo SG, Mikes F, Cabanelas JC, Paz-Abuin S, Baselga J (2003) Diffusion control on the cure kinetics of DGEBA with ethylenediamines. J Mater Process Technol 143-144:546-550

Quinti L, Allen N, Edge M, Murphy B, Perotti A (2003) A study of the luminescent complexes formed by the dye 1,4-dihydroxyanthraquinone (quinizarin) and Ga(III) and In(III). J Photochem Photobiol A 155:93-106

Roskowicz M, Smal T (2011) Durability of Belzona 1111 and Belzona 1812 adhesive composites. Polimery-W 56:471-477

Sasseville D (1998) Contact urticaria from epoxy resin and reactive diluents. Contact Dermatitis 38:57-58

Savko M, Kasackova P, Gbur P, Miskovsky P, Ulicny J (2007) Performance of Time Dependent Density Functional Theory on excitations of medium sized molecules - Test on ionic forms of anthraquinone dihydroxy derivatives. J Mol Struct (Theochem) 823:78-86

Say-Liang-Fat S, Cornard JP (2011) Al(III) complexation by alizarin studied by electronic spectroscopy and quantum chemical calculations. Polyhedron 30:2326-2332

Smith DA (2010) Metabolism, Pharmacokinetics and Toxicity of Functional Groups: Impact Of The Building Blocks Of Medicinal Chemsitry on ADMET. Royal Society of Chemistry, Cambridge

Soto J, Vázquez FJ, Yu A, Leache A, Quintanilla E (1989) Contact dermatitis by sensitization to amine-type antioxidants. Allergol Immunopathol (Madr) 17(5):263-265

Staneva D, Betcheva R, Cholvelon JM (2007) Optical sensor for aliphatic amines based on the simultaneous colorimetric and fluorescence responses of smart textile. J Appl Polym Sci 106:1950-1956

Tripathy R, Ojha U, Faust R (2011) Polyisobutylene Modified Bisphenol A Diglycidyl Ether Based Epoxy Resins Possessing Improved Mechanical Properties. Macromolecules 44:6800-6809

Wicks Z, Jones F, Pappas S, Wicks D (2007) Organic Coatings. Science and Technology. John Wiley and Sons, New York

doi:10.1186/2193-1801-2-593

Cite this article as: Jeliński et al: Application of alizarin colorimetric measurements for quantification of amine extraction by model food simulants from epoxy polymer. SpringerPlus 2013 2:593.

\section{Submit your manuscript to a SpringerOpen ${ }^{\circ}$ journal and benefit from:}

- Convenient online submission

- Rigorous peer review

- Immediate publication on acceptance

- Open access: articles freely available online

- High visibility within the field

- Retaining the copyright to your article

Submit your next manuscript at $\gg$ springeropen.com 